\title{
Math helps you wash a better hot bath
}

\section{Xianhao Teng}

School of Electric Power Engineering, North China Electric Power University, Baoding 071000, China.

18331136327@163.com

Keywords: Thermodynamics; Two-dimensional finite element; PDETOOL

\begin{abstract}
In this article, we apply thermodynamics and two-dimensional finite element to set up the temperature model and use PDETOOL in MATLAB to obtain the distribution of temperature of the water bath.

Firstly, based on the time and space,we formulated a model to account for the average temperature of the water bath and the flow rate of the water from taps. Then we analyze the two-dimensional finite element. We try to find out the factors that affect the temperature distribution, such as the position the faucet and shape of the bath tub.And we find that the oval bathtub is better than the rectangular tub in terms of water saving. We take the oval tub as an example, and get the temperature distribution and energy flow of the bath.
\end{abstract}

\section{Introduction}

Taking into account the manufacturing process and the limitations of the family space, we choose the main bathtub shape on the market.The circular bathtub,the oval bathtub and the rectangular tub are common.Through a simple market survey, the circular bathtub has been excluded because of its low market share.In the investigation of the main dimensions of the bathtub, the length of the bathtub must ensure the comfort of people lying.This paper assumes that it must be more than the length of body.

\section{Model}

\section{Question Analysis.}

We drew a long axis $1.5 \mathrm{~m}$ and short axis $0.8 \mathrm{~m}$ oval bathtub with the water inlet and the water outlet.Generally, the water outlet should be placed in the long axis, the water inlet can be based on the actual situation.We make use of the Matlab PDETOOL to scatter the upper surface of water into a finite small triangle.Each node is a vertex of a triangle.The temperature of the node is taken as an independent variable, and the default interpolation function is used in the PDETOOL toolbox.

\section{Model building and solving.}

According to the heat transfer equation, the bath water wall, air and people can be expressed as:

$$
\frac{\partial}{\partial x}\left(\lambda \frac{\partial T}{\partial x}\right)+\frac{\partial}{\partial y}\left(\lambda \frac{\partial T}{\partial y}\right)+\frac{\partial}{\partial z}\left(\lambda \frac{\partial T}{\partial z}\right)+q_{v}(x, y, z, t)-\rho C_{p} \frac{\partial T}{\partial t}=0
$$

Assuming that the water in the bathtub is the same in all directions, the heat transfer coefficient $\lambda$ is constant, the equation can be expressed as:

$$
\lambda\left(\frac{\partial^{2} T}{\partial x^{2}}+\frac{\partial^{2} T}{\partial y^{2}}+\frac{\partial^{2} T}{\partial z^{2}}\right)+q_{v}(x, y, z, t)-\rho C_{p} \frac{\partial T}{\partial t}=0
$$

Bath water temperature is kept constant, the heat of the hot water faucet is about equal. Without hot water, the bathtub can be regarded as a steady heat transfer. Then the equation can be expressed as:

$$
\frac{\partial^{2} T}{\partial x^{2}}+\frac{\partial^{2} T}{\partial y^{2}}+\frac{\partial^{2} T}{\partial z^{2}}+\frac{q_{v}(x, y, z, t)}{\lambda}=0
$$


In order to simplify, we focus on two-dimensional heat transfer, that is, from the top of the bath temperature and heat distribution.

Based on the two-dimensional plane, the equation is reduced to:

$$
\frac{\partial^{2} T}{\partial x^{2}}+\frac{\partial^{2} T}{\partial y^{2}}+\frac{q_{v}(x, y, t)}{\lambda}=0
$$

The above equation is two order partial differential equation, but also need to consider the water inlet and the water outlet, so it is difficult to get the accurate solution.In this paper, the finite element numerical simulation method of MATLAB is used.


Figure1. Distribution of bathtub with different shapes

The flow of hot water and the size of the bathtub will affect the water temperature distribution. The results are as follows Figure2,3,4:



Figure2. Original figure



Figure3. Change the flow of hot water 


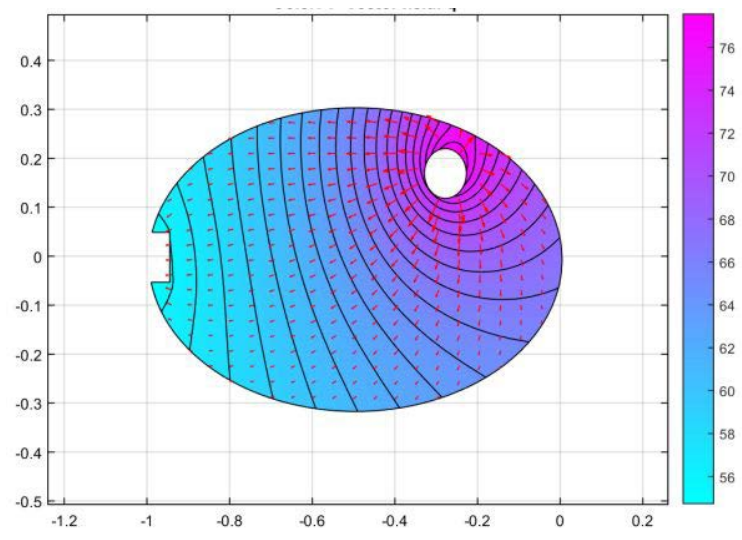

Figure4. Change the size of the bathtub

Figure 2 is as a reference. Figure 3 shows a lower heat flow, and we can find a corresponding decrease in the overall water temperature. Compared with Figure 2, Figure 4 indicates that the temperature is higher when the bath volume is reduced. When the water temperature and the volume of the bathtub are certain, the lower temperature is caused by the lower flow rate.

\section{Conclusions}

We can draw a conclusion from the above discussion, in order to achieve the purpose of saving hot water, the filling nozzle shall be located close to the bath center; when the water flow and the temperature from the hot water nozzle remains stable, considering the water and heat preservation, the oval bathtub is better than rectangular tub.when the size and comfortable bathtub are certain, volume is smaller, the flow rate is less.

\section{References}

[1] Khattiya Chalapat, Wichit Sritrakool, Spin-density functional approach to N-electron quantum dots within the Matlab pde-tool, Computational Materials Science, Volume 30, Issues 3-4, August 2004, Pages 288-295.

[2] D. De Santis, G. Geraci, A. Guardone, Equivalence conditions for the finite volume and finite element methods in spherical coordinates, Mathematics and Computers in Simulation, Volume 106, December 2014, Pages 60-75

[3] Jang Min Park, Martien A. Hulsen, Patrick D. Anderson, An extended finite element method for a diffuse-interface model, Journal of Computational and Applied Mathematics, Volume 272, 15 December 2014, Pages 25-40

[4] Tyler Van Buren, Edward Whalen, Michael Amitay et al.Vortex formation of a finite-span synthetic jet: High Reynolds numbers[J].Physics of fluids,2014,26(1):014101-1-014101-22.

[5] M. Baneshi.The Determination of Lithofacies Using an Optimized Neural Network and Well Log Data[J].Petroleum Science and Technology,2014,32(5/8):897-903. 